\title{
Remote sensing time series to assess fire recurrence patterns over Campos Amazônicos National Park
}

\author{
Daniel Borini Alves ${ }^{1,2 *}$, Fernando Pérez-Cabello ${ }^{2}$, Bruno Contursi Cambraia ${ }^{3}$
}

\footnotetext{
${ }^{1}$ Universidade Estadual Paulista (UNESP/Rio Claro), Rio Claro, Brasil.

${ }^{2}$ Universidade de Zaragoza (UNIZAR), Zaragoza, Espanha.

${ }^{3}$ Instituto Chico Mendes de Conservação da Biodiversidade (ICMBIO), Porto Velho, Brasil.

*e-mail: daniel.borini@unesp.br
}

\begin{abstract}
Fire is one of the major factors in the dynamics of terrestrial ecosystems, and its monitoring and assessment is very relevant for the understanding of ecological processes and human impacts at different scales. In this context, this work analyzes the fire incidence in the savannah enclave of Campos Amazônicos National Park (CANP), in the period 2000-2018, relating fire recurrence patterns with the current spatial distribution of tree cover physiognomies. Landsat multitemporal series were used to update the burned area mapping (2017 and 2018) through semi-automatic processes accompanied by visual supervision, complementing the burned area database that has been developed over the last years. Additionally, Landsat Tree Cover Continuous Field product was used to relate the different classes of fire recurrence and surface type. Over the past 19 years, 1.13 million hectares burned have been mapped, a total of 2.6 times the total size of the CANP enclave. Areas affected by fire between 2 and 4 times stand out, occupying 37.49\% of the enclave, and correspond to areas with predominance of physiognomies with medium-low and low percentage of tree cover. It was also observed that in areas with frequency higher than 4 fires there is a decrease in the tree cover percentage between the years 2000-2015, highlighting the prominent role of fire in maintaining the open phytosiognomies. Current research lines are focused on the analysis of fire experimental plots, using remote sensing data and fieldwork information to understand the relationships between fire and vegetation in the study area, providing information for the optimization of fire management strategies of CANP.
\end{abstract}

Keywords: burned areas, Landsat series, savannah enclave, Amazon 